Louisiana State University

LSU Digital Commons

$4-21-2008$

\title{
Reducing stray radiation dose to patients receiving passively scattered proton radiotherapy for prostate cancer
}

\author{
Phillip J. Taddei \\ University of Texas MD Anderson Cancer Center \\ Jonas D. Fontenot \\ University of Texas MD Anderson Cancer Center \\ Yuanshui Zheng \\ University of Texas MD Anderson Cancer Center \\ Dragan Mirkovic \\ University of Texas MD Anderson Cancer Center \\ Andrew K. Lee \\ University of Texas MD Anderson Cancer Center
}

See next page for additional authors

Follow this and additional works at: https://digitalcommons.Isu.edu/physics_astronomy_pubs

\section{Recommended Citation}

Taddei, P., Fontenot, J., Zheng, Y., Mirkovic, D., Lee, A., Titt, U., \& Newhauser, W. (2008). Reducing stray radiation dose to patients receiving passively scattered proton radiotherapy for prostate cancer. Physics in Medicine and Biology, 53 (8), 2131-2147. https://doi.org/10.1088/0031-9155/53/8/009

This Article is brought to you for free and open access by the Department of Physics \& Astronomy at LSU Digital Commons. It has been accepted for inclusion in Faculty Publications by an authorized administrator of LSU Digital Commons. For more information, please contact ir@lsu.edu. 


\section{Authors}

Phillip J. Taddei, Jonas D. Fontenot, Yuanshui Zheng, Dragan Mirkovic, Andrew K. Lee, Uwe Titt, and Wayne D. Newhauser 


\title{
Reducing stray radiation dose to patients receiving passively scattered proton radiotherapy for prostate cancer
}

\author{
Phillip J Taddei, Jonas D Fontenot, Yuanshui Zheng, Dragan Mirkovic, Andrew K Lee, Uwe \\ Titt, and Wayne D Newhauser \\ The University of Texas M. D. Anderson Cancer Center, 1515 Holcombe Blvd., Unit 94, Houston, \\ TX 77030, USA
}

Wayne D Newhauser: wnewhaus@mdanderson.org

\begin{abstract}
Proton beam radiotherapy exposes healthy tissue to stray radiation emanating from the treatment unit and secondary radiation produced within the patient. These exposures provide no known benefit and may increase a patient's risk of developing a radiogenic second cancer. The aim of this study was to explore strategies to reduce stray radiation dose to a patient receiving a 76 Gy proton beam treatment for cancer of the prostate. The whole-body effective dose from stray radiation, $E$, was estimated using detailed Monte Carlo simulations of a passively scattered proton treatment unit and an anthropomorphic phantom. The predicted value of $E$ was $567 \mathrm{mSv}$, of which $320 \mathrm{mSv}$ was attributed to leakage from the treatment unit; the remainder arose from scattered radiation that originated within the patient. Modest modifications of the treatment unit reduced $E$ by $212 \mathrm{mSv}$. Surprisingly, $E$ from a modified passive-scattering device was only slightly higher $(109 \mathrm{mSv})$ than from a nozzle with no leakage, e.g., that which may be approached with a spot-scanning technique. These results add to the body of evidence supporting the suitability of passively scattered proton beams for the treatment of prostate cancer, confirm that the effective dose from stray radiation was not excessive, and, importantly, show that it can be substantially reduced by modest enhancements to the treatment unit.
\end{abstract}

\section{Introduction}

Proton therapy is undergoing a rapid technological evolution of beam delivery equipment. Currently 25 centers worldwide are using protons to treat patients with cancer and other diseases, and 14 more facilities are planned or under construction (PTCOG, 2007). The main advantage of proton therapy over photon therapy is that it provides enhanced sparing of normal tissues from the primary beam in many clinical situations. However, the risks of late effects from stray radiation, e.g., neutrons and photons, are not yet fully understood (Hall, 2006), particularly those from neutrons. An improved understanding is needed for prostate treatments in particular because of the high and increasing incidence of prostate cancer and the large number of patients receiving proton therapy for that disease and because the stray radiation doses to these patients are expected to be among the largest of all proton treatments due to the high energy of the initial proton beam. 
Previous work has shown that the magnitude of stray radiation dose depends on the beam delivery technique and apparatus, the measurement location, the penetration depth, the field size, and the spread-out Bragg peak (SOBP) width (Mesoloras et al 2006, Zheng et al 2007). Measurements and simulations of equivalent dose and ambient dose equivalent from stray radiation per therapeutic dose $(H / D)$ for passively scattered proton beams have ranged from $<1 \mathrm{mSv} \mathrm{Gy}^{-1}$ to $80 \mathrm{mSv} \mathrm{Gy}^{-1}$ (cf Binns and Hough, 1997, Yan et al 2002, Polf et al 2005, Zheng et al 2007, 2008). For a spot-scanning beam of $177 \mathrm{MeV}$ protons, Schneider et al (2002) reported ambient dose equivalent from stray radiation that ranged from $0.9 \mathrm{mSv}$ $\mathrm{Gy}^{-1}$ to $37 \mathrm{mSv} \mathrm{Gy}^{-1}$, depending on the measurement location in a water phantom.

Additional investigations have predicted doses from stray radiation during treatment of specific anatomic structures. Agosteo et al (1998) estimated the absorbed dose from stray radiation per therapeutic absorbed dose for the treatment of deep tumors with a passively scattered beam delivery system to range between $0.1 \mathrm{mGy} \mathrm{Gy}^{-1}$ out of the field to as much as $17 \mathrm{mGy} \mathrm{Gy}^{-1}$ in the field. Jiang et al (2005) estimated whole-body effective doses from secondary neutrons per therapeutic proton dose of $2.3 \mathrm{mSv} \mathrm{Gy}^{-1}$ for lung tumors and 0.59 $\mathrm{mSv} \mathrm{Gy}^{-1}$ for paranasal sinus tumors, of which $36 \%$ and $16 \%$, respectively, were from stray radiation that originated within the patient. For proton treatments of the prostate, Fontenot $e t$ al (2008) found that for a prostate treatment using a passively scattered beam the wholebody effective dose from stray radiation per therapeutic absorbed dose was $7.8 \mathrm{mSv} \mathrm{Gy}^{-1}$ and the ambient dose equivalent per therapeutic absorbed dose at isocenter was $16 \mathrm{mSv}$ $\mathrm{Gy}^{-1}$. They reported that $40 \%$ was from stray radiation originating from within the patient. Although much effort has been made recently to quantify the magnitude of the stray radiation, little attention has been paid in the literature to reducing stray radiation doses through modifications of the treatment apparatus.

The aim of this work was to identify practical methods to reduce the whole-body effective dose from stray neutron radiation in a patient receiving a passively scattered proton therapy regimen for cancer of the prostate. In particular, the benefits of using a modified final collimator, local bulk shields and a supplemental upstream collimator were examined. The Monte Carlo simulation method was used to evaluate the performance of several such modifications to a contemporary, commercially available treatment unit.

\section{Methods}

\subsection{Treatment plan}

A treatment plan for a patient that was treated for prostate cancer at our institution was created using a commercial treatment planning system ${ }^{1}$ and kilovoltage CT images of the patient's pelvis. The plan used a lateral-opposed-pair field arrangement (Rossi et al 2004) with an absorbed dose of 76 Gy prescribed to $100 \%$ of the prostate. The prostate also served as the target volume used in treatment planning. The treatment beam had an energy of 250 $\mathrm{MeV}$ (range $=37.9 \mathrm{~cm}$ in water) as it entered the nozzle. The beam passed through a range modulator wheel (Koehler et al 1975) that yielded an SOBP of $11 \mathrm{~cm}$ width, a foil that laterally flattened and spread the beam to generate a $25.5 \mathrm{~cm}$ diameter uncollimated proton

\footnotetext{
${ }^{1}$ Eclipse Proton Planning, Varian Medical Systems, Inc., 3100 Hansen Way, Palo Alto, CA 94304.
} 
field, and an adjustable range shifter to produce a water-equivalent range at the surface of the patient of $27.5 \mathrm{~cm}$. As part of the planning process, a field-specific custom block made of brass was designed to collimate the treatment beam to cover the target volume. A fieldspecific custom range compensator (Wagner 1982) made of polymethyl methacrylate (PMMA) conformed the distal edge of the proton dose distribution to the target volume. For this treatment, a snout setting of a medium snout (i.e., up to $18 \times 18 \mathrm{~cm}^{2}$ field size) was selected. The air gap between the snout and the patient was minimal $(<5 \mathrm{~cm})$.

Because the treatment was approximately symmetrical about the patient's medial plane the two-field plan could be approximated as a single left-lateral beam, where the prescribed absorbed dose to the target volume (prostate) was maintained at $76 \mathrm{~Gy}$. The plan was exported for Monte Carlo simulation.

\subsection{Monte Carlo modeling of the treatment unit}

Monte Carlo simulations were performed using the Monte Carlo N-Particle eXtended (MCNPX) code version 2.6b (Pelowitz 2005) with parallel computing methods. The suitability of the MCNPX code for simulating therapeutic absorbed dose distributions and secondary radiation related to proton therapy has been established (Fontenot et al 2005, Herault et al 2005, Koch and Newhauser, 2005, Newhauser et al 2005, Polf and Newhauser, 2005, Polf et al 2005, Tayama et al 2006, Fontenot et al 2007b, Herault et al 2007, Newhauser et al 2007b, 2007a, Zheng et al 2007, 2008).

An MCNPX model of a commercial passive-scattering treatment unit ${ }^{2}$ (Newhauser et al 2007a) in service at The University of Texas MD Anderson Cancer Center (Smith et al 2003) was used in this work. The model used an elliptical proton source positioned immediately upstream of the nozzle, $3.29 \mathrm{~m}$ from isocenter, with a lateral Gaussian intensity distribution (horizontal FWHM $=5.4 \mathrm{~mm}$, vertical FWHM $=12.2 \mathrm{~mm}$ ), simulating the entrance of the parallel beam into the treatment head. The source was modeled with a Gaussian energy distribution with an initial mean energy of $250 \mathrm{MeV}$ (FWHM $=0.29$ $\mathrm{MeV}$ ). Within the nozzle, dual-scattering foils spread and flattened the beam laterally and a range-modulating wheel spread the Bragg peak in the direction of the beam axis. The model included beam monitors, range-shifting plates, structural and housing components (figure 1), and various static collimators in the treatment head. An $8 \mathrm{~cm}$ thick brass collimating aperture defined the final shape of the field. A field-specific range compensator conformed the treatment field to the distal surface of the target volume. The major features of the Monte Carlo model were described previously (Newhauser et al 2007a, Zheng et al 2007). However, the geometric model of the standard (unmodified) nozzle was extended in this work to investigate various strategies to reduce effective dose from stray radiation emanating from the nozzle as described in section 2.3.

\subsection{Modeling of the nozzle modifications}

Three strategies were tested to reduce neutron exposures: (1) adding bulk shields near the patient, (2) exchanging the final brass collimator with a tungsten-alloy replacement and (3)

${ }^{2}$ PROBEAT, Hitachi America, Ltd., 50 Prospect Avenue, Tarrytown, NY 10591. 
adding an upstream proton collimator near the range shifter assembly. The geometry of the supplemental bulk shields considered in this work is shown in figure 1, and the materials and dimensions are listed in table 1 . This shield comprised two layers; the first layer (item $\mathrm{E}$ in figure 1) was up to $29 \mathrm{~cm}$ thick, and the second layer (item F in figure 1) was $6 \mathrm{~cm}$ thick, totaling up to $35 \mathrm{~cm}$ of shielding. For the final collimator (item A in figure 1), brass was replaced with tungsten alloy, a commonly used material for multi-leaf collimators (cf Maughan et al 1994, Bues et al 2005, Pönisch et al 2006, Jang et al 2006, Tacke et al 2006, Farr et al 2006, Svensson et al 2007). The upstream collimator (item G in figure 1), also made of tungsten alloy, was located immediately downstream of the range-shifting plates (162 cm away from the surface of the patient and $186 \mathrm{~cm}$ upstream of isocenter). The aperture of this additional collimator had the same shape as the aperture of the final collimator, but its lateral dimensions were reduced to take into account the fact that it was closer to the virtual source (cf Newhauser et al 2007a). The lateral dimensions of the aperture were then expanded by $20 \%$ to minimize the effects of collimator-scattered protons on the dose distribution of the therapeutic proton beam (Titt et al 2008).

In this paper, stray radiation is defined as undesirable scattered radiation that is produced when the primary (therapeutic) proton beam interacts in the treatment unit or in the patient. The primary contributor to absorbed dose from stray radiation was secondary neutron radiation. Secondary neutrons were either generated in the patient or in the treatment unit. Neutrons generated in the patient, or 'internal neutrons', were considered a confounding factor in all simulations because they could not be attenuated by improvements to the nozzle. Therefore, to evaluate the performance of each strategy listed above, an ideal nozzle was modeled, in which all stray radiation that originated in the treatment unit was stopped immediately upstream of the patient. This was accomplished in MCNPX by placing a thin and wide cylindrical cell between the nozzle and the phantom with an importance of 0 for neutrons. The secondary radiation in the case of the ideal nozzle originated solely from interactions of primary protons inside the patient. Stated another way, by modeling an ideal nozzle, the lowest dose that could be achieved through modifications to the treatment unit was estimated. This model was also helpful in interpreting the relative importance of neutrons generated in the treatment unit, or 'external neutrons', and consequently the proposed modifications to the nozzle.

\subsection{Modeling of the patient}

Because the CT images used for the treatment plan were limited to the pelvic region, they were replaced in the simulations by a whole-body anatomical male phantom (Billings and Yucker, 1973) shown in figure 1. This phantom was previously adapted for use with the MCNPX code by Fontenot et al (2007a). For the purposes of this study, the phantom matched the size and shape of the patient. Absorbed dose and spectral fluence were sampled for protons, neutrons, photons and alpha particles in subvolumes of 12 organs of interest. This was accomplished by using twelve $2 \mathrm{~cm}$ diameter, spherical tally volumes, one in each of the brain, right lung, left lung, stomach, liver, colon, esophagus/thyroid, bladder, rectum, breast, gonads and prostate (isocenter). 
In each simulation, $10^{9}$ source protons were tracked along with secondary neutrons, photons, hydrogen and helium. The total computing time for all the simulations reported in this paper was 1061 cpu.days when run on $2.6 \mathrm{GHz}, 64$-bit processors (AMD Opteron) ${ }^{3}$.

\subsection{Reduction in effective dose}

For each nozzle modification, the resulting reduction in effective dose was given by

$$
\Delta E=E_{\mathrm{o}}-E, \quad(1)
$$

where $E_{\mathrm{o}}$ was the effective dose for the standard or unmodified nozzle and $E$ was the effective dose for the modified nozzle. The effective dose was separated into contributions from the primary (therapeutic) beam and the stray radiation (generated in the nozzle or patient):

$$
E=E_{\text {ther }}+E_{\text {stray }} .
$$

By substituting equation (2) into equation (1), the reduction in effective dose was

$$
\Delta E=\left(E_{\mathrm{o}, \text { ther }}+E_{\mathrm{o}, \text { stray }}\right)-\left(E_{\text {ther }}+E_{\text {stray }}\right) .
$$

In all cases, the dosimetric contributions from the therapeutic (primary) proton beam were constant by design. This included the organ absorbed doses, organ equivalent doses and the effective doses. Therefore, $E_{\mathrm{o} \text {,ther }}$ was by definition equal to $E_{\text {ther, }}$, and equation (3) was simplified to

$$
\Delta E=E_{\mathrm{o}, \text { stray }}-E_{\text {stray }}=\Delta E_{\text {stray }} .
$$

Thus, for each case, the reduction in effective dose was entirely attributable to a reduction in stray radiation. In the remainder of this work $\Delta E$ will denote the reduction in effective dose from stray radiation and $E$ will denote the effective dose from stray radiation. Next, the change in effective dose may be further broken down to distinguish the contributions of external and internal neutrons.

As stated above, the effective dose from stray radiation had two primary components, that from external neutrons and internal neutrons. Therefore, the effective dose was separated into contributions from internal neutrons originating in the patient, $E_{\mathrm{int}}$, and external neutrons generated in the nozzle, $E_{\text {ext }}$ :

$$
E_{\text {stray }}=E_{\text {ext }}+E_{\text {int }} .
$$

By substituting equation (5) into equation (4), the following is obtained:

\footnotetext{
${ }^{3}$ Advanced Micro Devices, Inc., One AMD Place, PO Box 3453, Sunnyvale, CA 95070.
} 


$$
\Delta E=\left(E_{\mathrm{o}, \mathrm{ext}}+E_{\mathrm{o}, \text { int }}\right)-\left(E_{\mathrm{ext}}+E_{\mathrm{int}}\right) .
$$

Just as $E_{\mathrm{o} \text {,ther }}$ was by definition equal to $E_{\text {ther }}$, because the properties of the therapeutic proton beam were held constant for each case, it followed that $E_{\mathrm{o}, \text { int }}$ was equal to $E_{\mathrm{int}}$. Equation (6) therefore could be simplified to

$$
\Delta E=E_{\mathrm{o}, \mathrm{ext}}-E_{\mathrm{ext}} .
$$

Thus, for each modified nozzle, the reduction in effective dose was entirely attributable to a reduction in external neutrons.

The performance of the shielding modifications was assessed using two figures of merit. The purpose of the first figure of merit, $E$, was to quantify the absolute effective dose from stray radiation for each particular modification of the standard nozzle. The second figure was simply the percentage reduction in the effective dose from external neutrons for each modification of the standard nozzle, or

$$
N=\frac{E_{\mathrm{o}, \mathrm{ext}}-E_{\text {ext }}}{E_{\mathrm{o}, \mathrm{ext}}} \times 100 \% .
$$

This figure of merit characterized how well the modified nozzle approached an ideal nozzle. For example, $N$ was $0 \%$ for the unmodified nozzle and $100 \%$ for an ideal nozzle, i.e. where external neutrons were completely eliminated. In equation (8), the numerator was determined from equation (7), and the denominator was calculated according to

$$
E_{\mathrm{o}, \mathrm{ext}}=E_{\mathrm{o}}-E_{\mathrm{o}, \mathrm{int}}, \quad(9
$$

where $E_{\mathrm{o}}$ was from a simulation of the unmodified nozzle (section 2.3) and where $E_{\mathrm{o}, \text { int }}$ was from a simulation of the ideal nozzle (section 2.3) in which all external neutrons were eliminated.

\subsection{Effective dose from stray radiation}

Following ICRP Publication 92 (ICRP, 2003), $E$ was calculated as the sum over specified organs or tissues, $T$,

$$
E=\sum_{T}\left(w_{T} H_{T}\right)
$$

where $w_{T}$ denotes the tissue weighting factor and $H_{T}$ denotes the organ equivalent dose from stray radiation. The values for $w_{T}$ were based on the recommendations from ICRP Publication 60 (ICRP 1991), and were intended to take into account the relative contribution to the risks of attributable detrimental effects. The values from ICRP Publication 60 were adjusted slightly as follows to take into account the large spatial variations of doses throughout the body from pelvic radiotherapy. The value of $w_{T}$ for lung was split between 
the right and left lungs, with $w_{T}=0.06$ for each. An average value of $H_{T}$ for the colon was calculated using data from the tally subvolumes of the colon and rectum, which was applied to the recommended $w_{T}=0.12$ for the colon. One tally subvolume was used to estimate the esophagus and thyroid equivalent doses; therefore, the $w_{T}$ values were combined to yield $w_{T}$ $=0.10$ for the subvolume that represented these organs. $w_{T}$ values for the breast, stomach, bladder, gonads, liver and brain were taken directly from ICRP Publication 60. The average of $H_{T}$ for all subvolumes was applied as an approximate equivalent dose to the remaining organs and tissues (e.g., red bone marrow, bone surface, skin, etc), and, together, these organs had a $w_{T}$ value of 0.19 . This follows the calculation approach from Fontenot et al (2008).

For each organ or tissue, $H_{T}$ was calculated according to

$$
H_{T}=\overline{w_{R}} D_{T}, \quad(11)
$$

where $D_{T}$ was the average absorbed dose from stray radiation in the organ or tissue and $\overline{w_{R}}$ was the mean radiation weighting factor used for all organs and all nozzle configurations as described below.

The org an-absorbed dose from stray radiation, $D_{T}$, was approximated by the absorbed dose calculated in the subvolume, as described above. The $D_{T}$ value was calculated as the sum of the absorbed dose from all major types of secondary radiation, including protons, $D_{T}(p)$; neutrons, $D_{T}(n)$; photons, $D_{T}(\gamma)$; and alpha particles, $D_{T}(\alpha)$. Energy transferred by these particles to electrons and recoil nuclei was absorbed locally at the interaction point and included in the appropriate tallies for absorbed dose. Thus,

$$
D_{T}=(76 \text { Gy }) \times \frac{D_{T}(p)+D_{T}(n)+D_{T}(\gamma)+D_{T}(\alpha)}{D_{\text {iso }}},
$$

where $D_{T}$ was normalized by the calculated absorbed dose to the isocenter subvolume, $D_{\text {iso }}$, located inside the prostate in the simulation and multiplied by the planned therapeutic dose of $76 \mathrm{~Gy}$.

An organ was classified as 'in-field' if absorbed dose calculated in the tally subvolume included a contribution from the primary treatment field. In-field organs with a non-zero $w_{T}$ included the bladder and the rectum, which had contributions to absorbed dose from primary protons. The remaining organs with tally subvolumes were classified as 'out-of-field' because the absorbed dose in their tally subvolumes resulted entirely from stray radiation.

In calculating $D_{T}$ using equation (12), the determination of the value of $D_{T}(p)$ for infield organs required special treatment because the MCNPX tallies of absorbed dose did not distinguish between therapeutic protons and secondary protons that originated from stray radiation. Specifically, the $D_{T}$ values for in-field organs were dominated by primary protons, whereas $D_{T}$ was defined as taking into account only stray radiation. Out-of-field organs did not require special treatment because primary protons did not contribute to absorbed dose to those organs. Also, distinguishing between therapeutic and stray radiation in out-of-field 
organs was not necessary for other radiation types since they were, by definition, all stray radiation. The methods for correcting $D_{T}(p)$ values for in-field organs are presented in the appendix.

A radiation weighting factor, $w_{R}$, was estimated for each organ and in each simulation based on the neutron spectral fluence incident on the tally subvolumes and following the recommendations of ICRP Publication 92 (ICRP 2003). This represents a slight departure from the methods recommended by the ICRP, in which $w_{R}$ is calculated based on the neutron fluence external to the body. This approach was developed for radiation protection applications involving irradiations of the whole body in a nearly uniform field. Instead, in this work $w_{R}$ was based on the neutron fields inside the body because much of the absorbed dose resulted from neutrons generated within the body. As will be shown in section 3, the values of $w_{R}$ for each organ varied only slightly. Therefore, for simplicity a mean $\overline{w_{R}}$ was used for all organs and all nozzle configurations except for the ideal nozzle.

\subsection{Uncertainty}

The uncertainties in $D_{T}, H_{T}, E$ and $\Delta E$ were estimated using standard propagation of uncertainties on the assumption that the component uncertainties were uncorrelated. Statistical uncertainties in the values of $D_{T}(X)$ were based on the coefficients of variation, $\sigma(X) / D_{T}(X)$, for the tallies of absorbed dose of each particle type $X$ calculated using MCNPX. The uncertainty in the value of $\overline{w_{R}}$ was set to zero because the variance was small compared to the implicit uncertainty in the definition of $w_{R}$, and the uncertainties in $w_{T}$ were assumed to be zero because these values were set by definition rather than measured within the study. The uncertainties were reported at the $68 \%$ confidence interval.

\section{Results}

\subsection{Effective dose from stray radiation}

Table 2 lists the values of effective dose from stray radiation, $E$, for the standard nozzle (with the brass final collimator and no supplemental shielding) (mod0), the standard nozzle with a tungsten-alloy final collimator $(\bmod 1)$, a modified nozzle with the optimal supplemental shielding determined by this study $(\bmod 2)$, the modified nozzle with supplemental shielding and a tungsten-alloy final collimator $(\bmod 3)$, the modified nozzle with supplemental shielding, a tungsten-alloy final collimator, and an additional upstream collimator (mod4), and the ideal nozzle (mod5). $E$ for the standard nozzle was $567 \mathrm{mSv}$. By replacing the standard nozzle with the ideal nozzle in the simulation, the reduction in $E$ was $320 \mathrm{mSv}$. This represented the maximum possible reduction in $E$ through improved nozzle design. This value was important because it provided a benchmark against which to compare the performance of each modification of the nozzle. It also represented the portion of the absolute effective dose that was due to external neutrons (56.4\%). The standard passivescattering nozzle resulted in a factor of 2.3 higher effective dose from stray radiation than what might be possible with a spot-scanning system.

Adding the dual-layered shielding to the standard nozzle $(\bmod 2)$ reduced $E$ by $57 \mathrm{mSv}$. By replacing the brass final collimator with one made of tungsten-alloy (mod3), $E$ was reduced by another $19 \mathrm{mSv}$. Finally, placing an extra tungsten-alloy collimator $186 \mathrm{~cm}$ upstream of 
isocenter (mod4) reduced $E$ by an additional $136 \mathrm{mSv}$ and did not alter the characteristics of the primary proton beam. Thus, modest modifications to the nozzle reduced the effective dose from stray radiation emanating from the treatment head by $212 \mathrm{mSv}$, from $567 \mathrm{mSv}$ to $355 \mathrm{mSv}$ (37.4\% reduction in $E$ ). This corresponded to an $N=66.3 \%$ reduction in effective dose from external neutrons.

The relative contribution to the risks of detrimental effects attributable to stray radiation was taken into account by multiplying the effective dose from stray radiation by the radiation weighting factor for each organ, $H_{T} w_{T}$. Values of $H_{T} w_{T}$ are plotted in figure 2 for the standard nozzle $(\bmod 0)$, the nozzle with supplemental shielding and a tungsten-alloy final collimator $(\bmod 3)$, the same modifications plus an extra upstream collimator $(\bmod 4)$ and the ideal nozzle (mod5). Contributions to risk were highest in the rectum, bladder, gonads and stomach. $H_{T} w_{T}$ was heavily influenced by the $w_{T}$ values, e.g., it was much higher for gonads than for bladder or rectum.

\subsection{Organ equivalent dose from stray radiation}

Figure 3(a) shows the values of equivalent dose from stray radiation for each organ, $H_{T}$, using these same four nozzle configurations. For the standard nozzle $(\bmod 0), H_{T}$ for each organ varied from $0.08 \mathrm{~Sv}$ to $1.5 \mathrm{~Sv}$, depending on the distance from the organ to isocenter. The rectum and bladder had the highest $H_{T}$ values at $1.5 \mathrm{~Sv}$ each. Even with the ideal nozzle (mod5) the rectum and bladder still received about $0.9 \mathrm{~Sv}$ of equivalent dose from internal neutrons. It should be noted that, because the bladder and rectum are adjacent to the prostate, the equivalent dose from primary protons was much larger than the equivalent dose from stray radiation.

Figure 3(b) shows the reduction in $H_{T}$ achieved after modifications were applied to the standard nozzle. Adding supplemental shielding and exchanging the brass final collimator with a tungsten-alloy replacement $(\bmod 3)$ reduced $H_{T}$ by less than $5 \%$ for the in-field organs. Because of the shape of the shielding (as shown in figure 1), the available space for supplemental shielding was smallest for the in-field organs. Supplemental local shielding clearly reduced $H_{T}$ for the out-of-field organs because the shielding thickened with off-axis distance. Organs farther from isocenter were largely spared from stray radiation when shielding was added.

When the steel and PMMA layers of shielding were added, the final collimator material was changed to tungsten alloy, and an upstream collimator was included (mod4), $H_{T}$ was reduced for all organs. The organs that were partially in the proton field (i.e., rectum and bladder) had a marked reduction in $H_{T}$, approximately $18 \%$. Organs that were characterized as out-of-field but were within $35 \mathrm{~cm}$ of isocenter (i.e., gonads, colon, liver and stomach) had even larger percentage reductions in $H_{T}$ (33\% to $72 \%$ ). For organs more than $35 \mathrm{~cm}$ from isocenter (i.e., breast, lungs, esophagus, thyroid and brain), $H_{T}$ was reduced by more than $72 \%$.

For all tally subvolumes (including isocenter) and all nozzle configurations, excluding the ideal nozzle, the mean value $\overline{w_{R}}$ was $5.9 \pm 0.2$. The maximum value of $w_{R}$ was 7.38 and the minimum value was 4.55 . 


\subsection{Local bulk shielding selection}

Six configurations of supplemental shielding were tested, with one case having two layers of different materials (as shown in figure 1). For each case, $\Delta E$ is shown in figure 4 . For lowdensity shielding materials, such as PMMA, polyethylene and concrete, relatively small $\Delta E$ values were observed. In the case of polyethylene, adding boron as a thermal neutron absorber to the material composition did not significantly reduce $E$ because thermal neutrons contributed only a small proportion to $H_{T}$ (Yan et al 2002, Zheng et al 2008). When relatively high-density steel was used for the first layer of supplemental shielding, $E$ was reduced considerably more than when PMMA was used for both layers. Also, a layered configuration of high-density steel (density $=7.9 \mathrm{~g} \mathrm{~cm}^{-3}$ ) and hydrogenous PMMA ( $8 \%$ hydrogen by mass) reduced $E$ slightly more than high-density material alone. Because it had the largest $\Delta E$ and appeared to be practicable, the dual-layered shielding design, with highdensity steel as the first layer and hydrogenous PMMA as the second layer, was selected to be analyzed further in the study $(\bmod 2)$.

\section{Discussion}

Monte Carlo simulations suggest that it may be possible to reduce the effective dose from stray radiation emanating from the treatment unit, or external neutrons, by approximately $66 \%$ for patients receiving passively scattered proton therapy for prostate cancer. In the simulations, this corresponded to a $37 \%$ reduction in the absolute effective dose from stray radiation originating in the treatment unit and the patient. This result approaches what may be achieved through alternative nozzle designs, for example a nozzle that implements a spotscanning technique. The reduction was achieved with the following modest modifications, listed in descending order of effectiveness: (1) adding a proton collimator far upstream from the patient, (2) using tungsten alloy in place of brass for the final collimator and (3) increasing local shielding near the patient. The results revealed that the performance of supplemental local shielding can be increased by using a high-density layer along with a hydrogenous layer.

The effective dose from stray radiation per therapeutic absorbed dose, $E / D$, was similar to that reported in previous studies. The predicted $E / D$ value for the standard nozzle was 9.2 $\mathrm{mSv} \mathrm{Gy}{ }^{-1}$. This is in good agreement with previous $H / D$ determinations made in the presence of a phantom. Specifically, measured $H / D$ values from Yan et al (2002) and simulated $H / D$ values from Polf et al (2005) and Zheng et al (2008) ranged from $1 \mathrm{mSv}$ $\mathrm{Gy}^{-1}$ to $15 \mathrm{mSv} \mathrm{Gy}^{-1}$, depending on the location and other variables.

In this study, the component of $E / D$ that was from external neutrons was $5.2 \mathrm{mSv} \mathrm{Gy}^{-1}$. This value is consistent with the $H / D$ values calculated by Zheng et al (2007) for a $250 \mathrm{MeV}$ beam without a phantom present, which ranged from $1 \mathrm{mSv} \mathrm{Gy}^{-1}$ to $20 \mathrm{mSv} \mathrm{Gy}^{-1}$ within $120 \mathrm{~cm}$ of isocenter. This value was less than the measured $H / D$ values reported by Binns and Hough (1997) for a pre-clinical nozzle, which were between $33 \mathrm{mSv} \mathrm{Gy}{ }^{-1}$ and $80 \mathrm{mSv}$ $\mathrm{Gy}^{-1}$. However, the results from Binns and Hough are the highest $H / D$ values in the literature, and the differences with respect to the present work are likely due to the considerable differences in the treatment units. 
It was determined that $43 \%$ of $E / D$ for the standard nozzle was from internal neutrons. This result is similar to the effective dose from internal neutrons that was reported by Fontenot $e t$ al (2008) for a prostate treatment (40\%) and by Jiang et al (2005) for a lung treatment $(36 \%)$.

The recommended risk coefficient of the NCRP (1997) for low-dose-rate, exposure-induced death for middle-aged men is $4.2 \%$ per Sv. Applying this risk coefficient to the reduction in effective dose from stray radiation in this study, the adoption of the three-part strategy described here (i.e., additional upstream collimator, supplemental bulk shielding and a final collimator of tungsten alloy) would result in approximately 9 fewer second cancer fatalities per 1000 patients undergoing passively scattered proton therapy for prostate cancer.

The scope of this study was to investigate whether simple methods could be used to reduce stray radiation exposure for patients undergoing proton therapy for prostate cancer. The results of this study suggest that such modifications are feasible and effective. In addition, it appears likely that the effectiveness and compactness of these enhancements may be substantially optimized relative to the nozzle designs unique to each proton therapy facility.

Existing facilities for high-energy neutron therapy may serve as models for designing future passively scattered proton treatment units to minimize stray radiation exposure. For example, the most promising modification to the nozzle, and potentially the simplest and least expensive, was the addition of a field-specific pre-collimator located far upstream from the patient. One suggestion might be to implement this strategy using a computer-controlled multi-leaf collimator fashioned after the multi-leaf collimators currently being used with intensity-modulated radiation therapy (Bues et al 2005, Pönisch et al 2006, Jang et al 2006, Tacke et al 2006), as is already being done at some facilities. For instance, the neutron therapy facility at the University of Washington employs a computer-controlled steel and polyethylene multi-leaf collimator (Brahme et al 1983). The use of both a manually controlled tungsten multi-rod collimator and a computer-controlled steel multi-leaf collimator for attenuating fast neutrons was investigated at the Harper Hospital in Detroit, MI (Maughan et al 2001, Farr et al 2006); it was determined that both steel and tungsten are suitable materials for fast neutron attenuation and had acceptable activation levels (the former is cheaper, but the latter has a higher linear attenuation coefficient). A passively scattered nozzle at the Proton Medical Research Center at the University of Tsukuba (Japan) uses a brass multi-leaf collimator to remove non-therapeutic protons from the beam. The spot-beam-scanning system at the National Institute of Radiological Sciences in Chiba, Japan uses a backup multi-rod collimator made of brass (Tsunemoto et al 1985).

Reinforcing the idea of incorporating a variable multi-leaf collimator in nozzle design is the work by Tayama et al (2006), who investigated the effect of changing the aperture size of a pre-collimator on dose equivalent from stray neutrons emanating from the nozzle. By changing the aperture from $220 \mathrm{~mm}$ to $54 \mathrm{~mm}$, the $H / D$ value was reduced by more than $40 \%$ for locations within $80 \mathrm{~cm}$ trans-axially of isocenter. This evidence further suggests that adding an adjustable pre-collimator far upstream of the patient may be critical in reducing stray radiation dose to patients undergoing proton therapy. This pre-collimator would serve to both stop non-therapeutic protons and move the effective neutron source to a greater distance from the patient. 
The types of materials used for these modifications may also be optimized for each facility. Neutron therapy facilities have used pressed wood (Bonnett et al 1980) and iron (Maruyama et al 1978) to moderate and attenuate high-energy neutrons. Osmium has also been suggested as a collimator material for light ion beams (Svensson et al 2007). The Monte Carlo simulations performed in this work may serve as a model for optimizing shielding materials for specific facilities.

The present study had some limitations. In particular, one factor that may have introduced systematic error was the method for estimating dose from secondary protons for organs, where the total dose was dominated by primary protons (described in the appendix). The correction for determining absorbed dose from stray radiation for the bladder and rectum introduced uncertainties that were very difficult to quantify. However, since the combined $w_{T}$ for these organs was only 0.11 , this approximation was of minor importance since it had minimal effects on the predicted $\Delta E$ values.

There was also inherent uncertainty in applying the physical dosimetric quantities to estimate the risk of biological effects. The definition of $w_{R}$ used in this study was applied to fast and high energy neutrons, but at present the relative biological effectiveness of these neutrons is uncertain. For neutron-induced carcinogenesis, relative biological effectiveness may be as much as 50 (Wolf et al 2000), 100 (Shellabarger et al 1980) or more than 100 (Kellerer et al 2006) at low doses. If the values of $w_{R}$ were modified, the quantities of effective dose would scale linearly with the value of $w_{R}$. However, since the second figure of merit of this study was reported in terms of the percent reduction in effective dose using a constant $\overline{w_{R}}$, it would remain the same at different $w_{R}$ values. Therefore, this is not a serious limitation.

In conclusion, this Monte Carlo study establishes the viability of implementing modest modifications to a standard passively scattered proton treatment unit in order to reduce markedly the whole-body effective dose from stray radiation in a patient receiving proton radiotherapy for prostate cancer.

\section{Acknowledgments}

This work was supported in part by a research grant from Varian Medical Systems (Palo Alto, CA). The authors wish to thank Kathryn Carnes for her assistance in preparing this paper.

\section{Appendix. Approximation for DT(p) of in-field organs}

For out-of-field organs, the values of $D_{T}(X)$ for each particle type $X$ were assumed to be entirely from stray radiation. For in-field organs, this assumption was not valid because primary, therapeutic protons had a much larger contribution to $D_{T}(p)$ than protons produced from stray radiation. Therefore, for in-field organs, an alternative approach was used to determine $D_{T}(p)$.

For a constant $\overline{w_{R}}$ applied to each $D_{T}$ and a constant set of values of $w_{T}$ applied in each configuration, equations (3), (10) and (11) in the main text were combined to derive 


$$
\Delta E=\sum_{T} w_{T} \overline{w_{R}}\left(D_{T, \mathrm{o}, \text { ther }}+D_{T, \mathrm{o}, \mathrm{stray}}-D_{T, i, \mathrm{ther}}-D_{T, i, \mathrm{stary}}\right)=\sum_{T} w_{T} \overline{w_{R}} \Delta D_{T, i}
$$

where $\Delta D_{T, i}$ was the change in absorbed dose to an organ or tissue for each nozzle configuration, $i$. The relative uncertainty in $\Delta D_{T, i}$ was

$$
\frac{\sigma_{\Delta D_{T, i}}}{\Delta D_{T, i}}=\frac{\sqrt{\sigma_{D_{T, \mathrm{o}, \text { ther }}^{2}}^{2}+\sigma_{D_{T, \mathrm{o}, \text { stary }}}^{2}+\sigma_{D_{T, i, \text { ther }}}^{2}+\sigma_{D_{T, i, \text { stary }}}^{2}}}{D_{T, \mathrm{o}, \text { ther }}+D_{T, \mathrm{o}, \text { stary }}-D_{T, i, \text { ther }}-D_{T, i, \mathrm{stary}}} .
$$

For the out-of-field organs, $\frac{\sigma_{\Delta D_{T, i}}}{\Delta D_{T, i}}$ was small $(<0.10)$. For in-field organs, however, just as $D_{T, 0, \text { ther }}$ and $D_{T, i, \text { ther }}$ were much larger than $D_{T, \mathrm{o}, \text { stray }}$ and $D_{T, i, \text { stray }}, \sigma \Delta D_{T, \mathrm{o} \text {, ther }}$ and $\sigma \Delta D_{T, i \text {, ther }}$ were much larger than $\sigma \Delta D_{T, \mathrm{o} \text {,stay }}$ and $\sigma \Delta D_{T, i, \text { stray }}$. As a result, although the large values of therapeutic dose canceled in the denominator, their large uncertainties were added in the numerator, causing $\frac{\sigma_{\Delta D_{T, i}}}{\Delta D_{T, i}}$ to be large. This large coefficient of variance would have been propagated to higher-order quantities of $H_{T}, E$ and $\Delta E$.

Therefore, an alternative approach was developed to provide reasonably small uncertainties in absorbed dose for the in-field organs. Applying equation (4) of the main text, the values for therapeutic absorbed dose and their uncertainties were assumed to be zero, and the absorbed dose tallies for neutrons, photons and alpha particles were all assumed to be from stray radiation. The absorbed dose tally for protons in MCNPX did not distinguish between primary and secondary protons. Therefore, the contribution to absorbed dose from primary and secondary protons was not separated, and $D_{T}(p)$ was not obtained from the tally. Instead, the following methods were used to determine $D_{T}(p)$.

The values of $D_{T}(p) / D_{T}(n)$ were analyzed for all out-of-field organs and all configurations, excluding the ideal nozzle. The analysis showed that $D_{T}(p) / D_{T}(n)$ was independent of the distance of the organ from isocenter (data not shown). The mean of the ratio of absorbed dose from secondary protons to that from secondary neutrons, $A$, was given by

$$
A=\frac{1}{9} \sum_{i=1}^{9}\left[\frac{1}{9} \sum_{T=1}^{9} \frac{D_{T}(p)}{D_{T}(n)}\right]
$$

and was determined for the nine out-of-field organs, $T$, in the nine nozzle configurations, $i$, excluding the ideal nozzle. For the in-field organs, the absorbed dose from stray proton radiation was approximated as

$$
D_{T}(p)=A \cdot D_{T}(n)
$$

Thus, for the in-field organs, equation (12) became 


$$
D_{T}=(76 \mathrm{~Gy}) \times \frac{A \cdot D_{T}(\mathrm{n})+D_{T}(\mathrm{n})+D_{T}(\gamma)+D_{T}(\alpha)}{D_{\text {iso }}} .
$$

This approach ensured that $D_{T}$ included only absorbed dose from stray radiation. The resulting value of $A$ was $5.7 \pm 0.2$.

\section{References}

Agosteo S, Birattari C, Caravaggio M, Silari M, Tosi G. Secondary neutron and photon dose in proton therapy. Radiother Oncol. 1998; 48:293-305. [PubMed: 9925249]

Billings, MP.; Yucker, WR. Summary final report: the computerized anatomical man (cam) model Report MDC G4655. Huntington Beach, CA: McDonnell Douglas Astronautics Company; 1973.

Binns PJ, Hough JH. Secondary dose exposures during $200 \mathrm{MeV}$ proton therapy. Radiat Prot Dosim. 1997; 70:441-4.

Bonnett DE, Williams JR, Parnell CJ. The isocentric fast neutron therapy facility at Edinburgh. Brit J Radiol. 1980; 53:12-20. [PubMed: 6766336]

Brahme A, Eenmaa J, Lindback S, Montelius A, Wootton P. Neutron beam characteristics from 50 $\mathrm{MeV}$ protons on beryllium using a continuously variable multi-leaf collimator. Radiother Oncol. 1983; 1:65-76. [PubMed: 6438702]

Bues M, Newhauser WD, Titt U, Smith AR. Therapeutic step and shoot proton beam spot-scanning with a multi-leaf collimator: a Monte Carlo study. Radiat Prot Dosim. 2005; 115:164-9.

Farr JB, Maughan RL, Yudelev M, Blosser E, Brandon J, Horste T, Forman JD. Compact multileaf collimator for conformal and intensity modulated fast neutron therapy: electromechanical design and validation. Med Phys. 2006; 33:3313-20. [PubMed: 17022226]

Fontenot JD, Newhauser WD, Bloch C, White RA, Titt U, Starkschall G. Determination of output factors for small proton therapy fields. Med Phys. 2007a; 34:489-98. [PubMed: 17388166]

Fontenot JD, Newhauser WD, Titt U. Design tools for proton therapy nozzles based on the doublescattering foil technique. Radiat Prot Dosim. 2005; 116:211-5.

Fontenot J, Taddei P, Zheng Y, Mirkovic D, Jordan T, Newhauser WD. Equivalent dose and effective dose from stray radiation during passively-scattered proton radiotherapy for prostate cancer. Phys Med Biol. 2008; 53:1677-88. [PubMed: 18367796]

Fontenot, JD.; Zheng, YZ.; Taddei, P.; Newhauser, W. Stray radiation exposure during proton radiotherapy of the prostate: the influence of the patient on scatter and production. Med Phys; Oral Presentation 49th Annual Meeting of the American Association of Physicists in Medicine; 2007b. p. 2507

Hall EJ. Intensity-modulated radiation therapy, protons, and the risk of second cancers. Int J Radiat Oncol Biol Phys. 2006; 65:1-7. [PubMed: 16618572]

Herault J, Iborra N, Serrano B, Chauvel P. Monte Carlo simulation of a protontherapy platform devoted to ocular melanoma. Med Phys. 2005; 32:910-9. [PubMed: 15895573]

Herault J, Iborra N, Serrano B, Chauvel P. Spread-out Bragg peak and monitor units calculation with the Monte Carlo code MCNPX. Med Phys. 2007; 34:680-8. [PubMed: 17388186]

ICRP. Ann ICRP. Vol. 21. New York: Pergamon Press; 1991. Recommendations of the International Commission on Radiological Protection: publication 60.

ICRP. Ann ICRP. Vol. 33. New York: Pergamon Press; 2003. Relative Biological effectiveness (RBE), quality factor $(\mathrm{Q})$, and radiation weighting factor $\left(w_{R}\right)$ : publication 92; p. 1-121.

Jang SY, Vassiliev ON, Liu HH, Mohan R, Siebers J. Development and commissioning of a multileaf collimator model in Monte Carlo dose calculations for intensity-modulated radiation therapy. Med Phys. 2006; 33:770-81. [PubMed: 16878579]

Jiang H, Wang B, Xu XG, Suit HD, Paganetti H. Simulation of organ-specific patient effective dose due to secondary neutrons in proton radiation treatment. Phys Med Biol. 2005; 50:4337-53. [PubMed: 16148397] 
Kellerer AM, Ruhm W, Walsh L. Indications of the neutron effect contribution in the solid cancer data of the A-bomb survivors. Health Phys. 2006; 90:554-64. [PubMed: 16691103]

Koch N, Newhauser W. Virtual commissioning of a treatment planning system for proton therapy of ocular cancers. Radiat Prot Dosim. 2005; 115:159-63.

Koehler AM, Schneider RJ, Sisterson JM. Range modulators for protons and heavy ions. Nucl Instrum Methods. 1975; 131:437-40.

Maruyama T, Inada T, Hiraoka T, Kawachi K, Hashizume T, Tsunemoto H, Kutsutani Y, Umegaki Y. Design and evaluation of a semicontinuously variable collimator for a cyclotron neutron radiotherapy. Nippon Acta Radiol. 1978; 38:633-42. [PubMed: 704296]

Maughan RL, Powers WE, Blosser HG. A superconducting cyclotron for neutron radiation therapy. Med Phys. 1994; 21:779-85. [PubMed: 7935214]

Maughan RL, Yudelev M, Forman JD, Williams SB, Gries D, Fletcher TM, Chapman W, Blosser EJ, Horste T. Attenuation and activation characteristics of steel and tungsten and the suitability of these materials for use in a fast neutron multileaf collimator. Med Phys. 2001; 28:1006-9. [PubMed: 11439469]

Mesoloras G, Sandison GA, Stewart RD, Farr JB, Hsi WC. Neutron scattered dose equivalent to a fetus from proton radiotherapy of the mother. Med Phys. 2006; 33:2479-90. [PubMed: 16898451]

Moyers MF, Coutrakon GB, Ghebremedhin A, Shahnazi K, Koss P, Sanders E. Calibration of a proton beam energy monitor. Med Phys. 2007; 34:1952-66. [PubMed: 17654898]

NCRP. Report Number 126. Bethesda, MD: National Council on Radiation Protection and Measurements; 1997. Uncertainties in fatal cancer risk estimates used in radiation protection.

Newhauser W, Fontenot J, Zheng Y, Polf J, Titt U, Koch N, Zhang X, Mohan R. Monte Carlo simulations for configuring and testing an analytical proton dose-calculation algorithm. Phys Med Biol. 2007a; 52:4569-84. [PubMed: 17634651]

Newhauser WD, Koch NC, Fontenot JD, Rosenthal SJ, G DS, Fitzek MM, Mohan R. Dosimetric impact of tantalum markers used in the treatment of uveal melanoma with proton beam therapy. Phys Med Biol. 2007b; 52:3979-90. [PubMed: 17664589]

Newhauser W, Koch N, Hummel S, Ziegler M, Titt U. Monte Carlo simulations of a nozzle for the treatment of ocular tumours with high-energy proton beams. Phys Med Biol. 2005; 50:5229-49. [PubMed: 16264250]

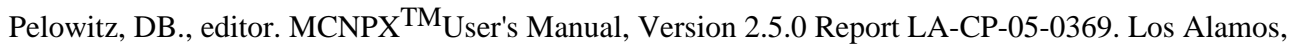
NM: Los Alamos National Laboratory; 2005.

Polf JC, Newhauser WD. Calculations of neutron dose equivalent exposures from range-modulated proton therapy beams. Phys Med Biol. 2005; 50:3859-73. [PubMed: 16077232]

Polf JC, Newhauser WD, Titt U. Patient neutron dose equivalent exposures outside of the proton therapy treatment field. Radiat Prot Dosim. 2005; 115:154-8.

Pönisch F, Titt U, Kry SF, Vassiliev ON, Mohan R. MCNPX simulation of a multileaf collimator. Med Phys. 2006; 33:402-4. [PubMed: 16532947]

PTCOG. Particle Therapy Co-Operative Group. 2007 Sep. website http://ptcog.web.psi.ch/ ptcentres.html

Rossi CJ Jr, Slater JD, Yonemoto LT, Jabola BR, Bush DA, Levy RP, Grove R, Slater JM. Influence of patient age on biochemical freedom from disease in patients undergoing conformal proton radiotherapy of organ-confined prostate cancer. Urology. 2004; 64:729-32. [PubMed: 15491710]

Schneider U, Agosteo S, Pedroni E, Besserer J. Secondary neutron dose during proton therapy using spot scanning. Int J Radiat Oncol Biol Phys. 2002; 53:244-51. [PubMed: 12007965]

Shellabarger CJ, Chmelevsky D, Kellerer AM. Induction of mammary neoplasms in the SpragueDawley rat by $430 \mathrm{keV}$ neutrons and x-rays. J Natl Cancer Inst. 1980; 64:821-33. [PubMed: 6928995]

Smith, A.; Newhauser, W.; Ltinkic, M.; Hay, A.; McMaken, B.; Styles, J.; Cox, J. The University of Texas MD Anderson Cancer Center Proton Therapy Facility. In: Duggan, JL.; Morgan, IL., editors. Application of Accelerators in Research and Industry: $17^{\text {th }}$ Int Conf. 2003. p. 1073-6.

Svensson R, Larsson S, Gudowska I, Holmberg R, Brahme A. Design of a fast multileaf collimator for radiobiological optimized IMRT with scanned beamsof photons, electrons, and light ions. Med Phys. 2007; 34:877-88. [PubMed: 17441233] 
Tacke MB, Szymanowski H, Oelfke U, Schulze C, Nuss S, Wehrwein E, Leidenberger S. Assessment of a new multileaf collimator concept using GEANT4 Monte Carlo simulations. Med Phys. 2006; 33:1125-32. [PubMed: 16696490]

Tayama R, Fujita Y, Tadokoro M, Fujimaki H, Sakae T, Terunuma T. Measurement of neutron dose distribution for a passive scattering nozzle at the Proton Medical Research Center (PMRC). Nucl Instrum Methods A. 2006; 564:532-6.

Titt U, Zheng Y, Vassiliev ON, Newhauser WD. Monte Carlo investigation of collimator scatter of proton-therapy beams produced using the passive scattering method. Phys Med Biol. 2008; 53:487-504. [PubMed: 18185001]

Tsunemoto H, Morita S, Ishikawa T, Furukawa S, Kawachi K, Kanai T, Ohara H, Kitagawa T, Inada T. Proton therapy in Japan. Radiat Res Suppl. 1985; 8:S235-43. [PubMed: 3003785]

Wagner MS. Automated range compensation for proton therapy. Med Phys. 1982; 9:749-52. [PubMed: 6296648]

Wolf C, Lafuma J, Masse R, Morin M, Kellerer AM. Neutron RBE for induction of tumors with high lethality in Sprague-Dawley rats. Radiat Res. 2000; 154:412-20. [PubMed: 11023605]

Yan X, Titt U, Koehler AM, Newhauser WD. Measurement of neutron dose equivalent to proton therapy patients outside of the proton radiation field. Nucl Instrum Methods A. 2002; 476:429-34.

Zheng Y, Fontenot J, Taddei P, Mirkovic D, Newhauser W. Monte Carlo simulations of neutron spectral fluence, radiation weighting factor and ambient dose equivalent for a passively scattered proton therapy unit. Phys Med Biol. 2008; 53:187-201. [PubMed: 18182696]

Zheng Y, Newhauser W, Fontenot J, Taddei P, Mohan R. Monte Carlo study of neutron dose equivalent during passive scattering proton therapy. Phys Med Biol. 2007; 52:4481-96. [PubMed: 17634645] 


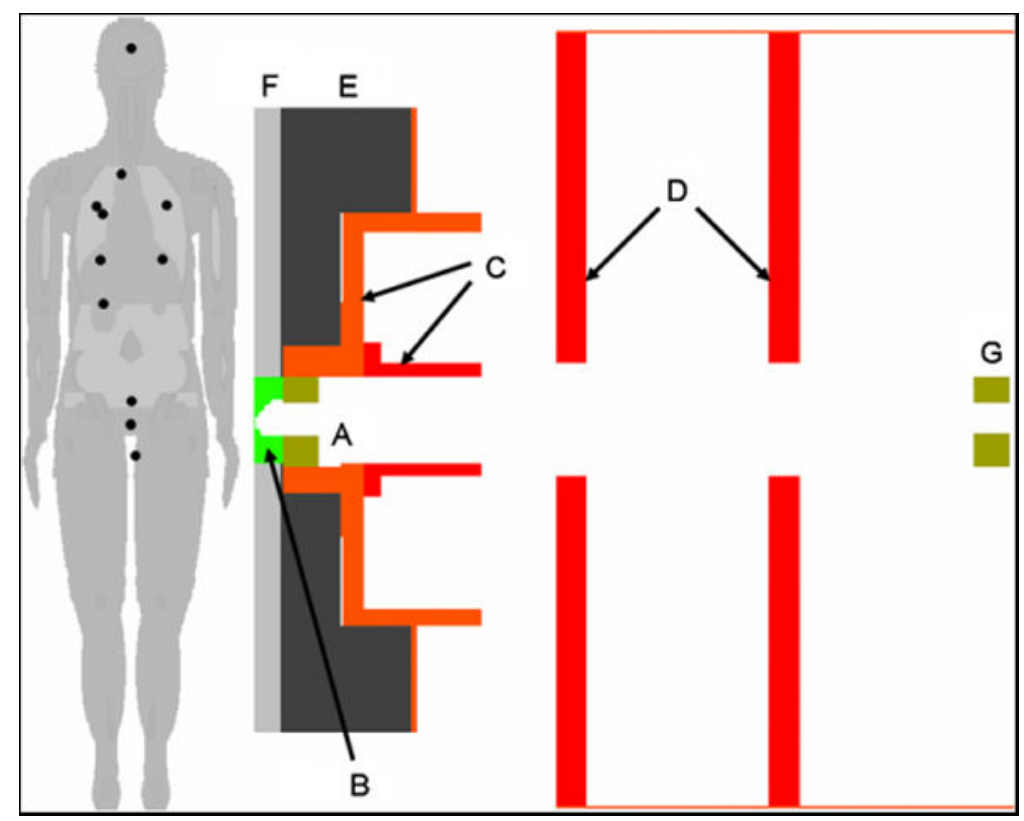

Figure 1.

Anatomical models of a man in the treatment position and the treatment nozzle. The portion of the treatment head near the patient shows the final collimator (A), range compensator (B), snout (C) and other collimators (D). The phantom was arranged to represent the patient in the treatment position. Shielding layers one (E) and two $(F)$ were added between the nozzle and the patient. An additional tungsten-alloy pre-collimator $(\mathrm{G})$ in the same shape as the final collimator but appropriately scaled in the lateral dimensions was placed $186 \mathrm{~cm}$ upstream of isocenter. The projections of the locations of the tally subvolumes onto the displayed plane are shown as black circles on the phantom. 


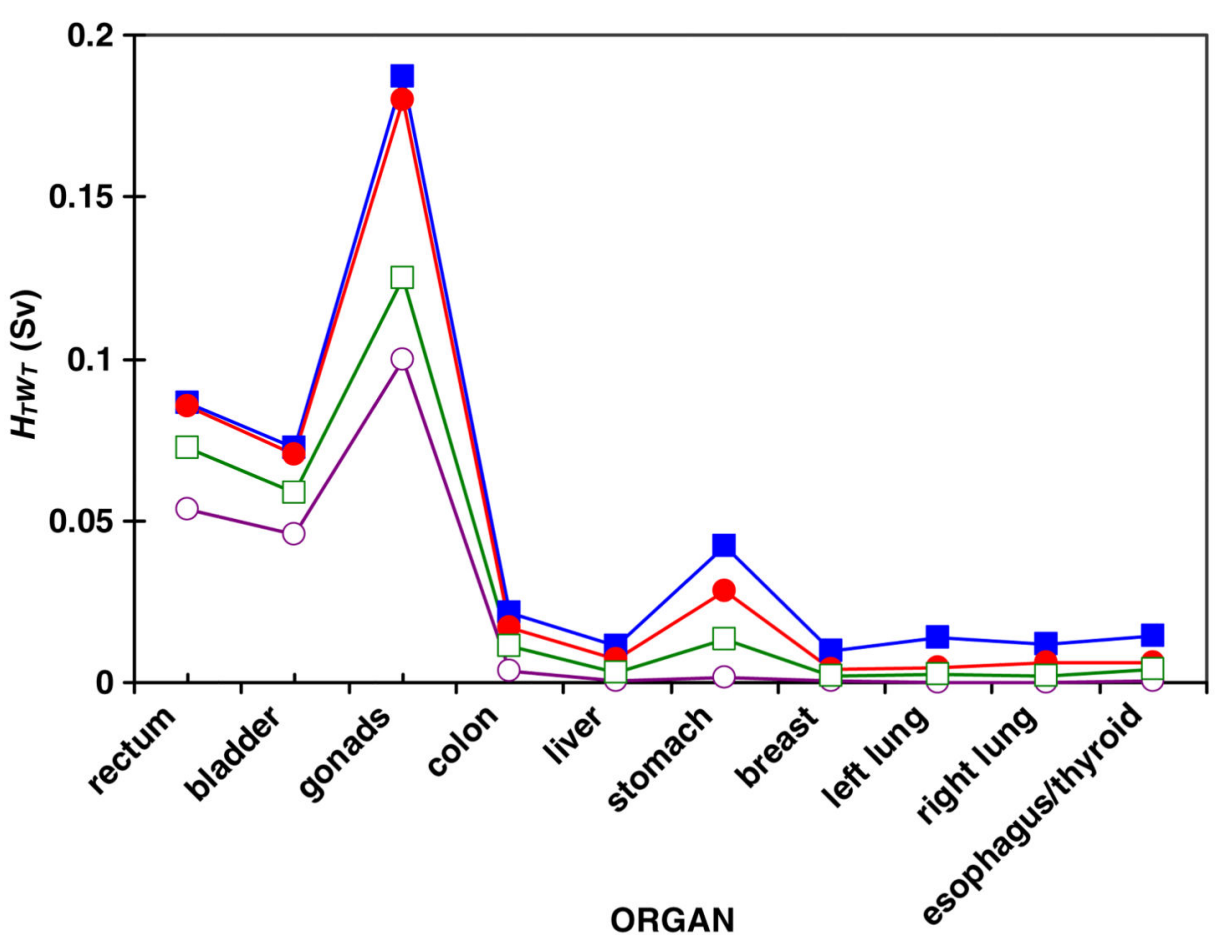

Figure 2.

The product of the equivalent dose from stray radiation and the tissue weighting factor, $H_{T}$ $w_{T}$, for each organ in simulations of the standard nozzle (mod0) (solid squares), a nozzle equipped with supplemental shielding and a tungsten-alloy final collimator (mod3) (solid circles), the equipped nozzle with an additional collimator far upstream of the patient (mod4) (open squares) and the ideal nozzle (mod5) (open circles). The values represent the relative contribution to effective dose and risks of detrimental effects. The lines were added to guide the eye. Distance from the organ to isocenter increases from left to right on the plot. These data were for a 76 Gy SOBP proton treatment of the prostate. Statistical uncertainties in values of $H_{T} w_{T}$ are contained within the data markers. 

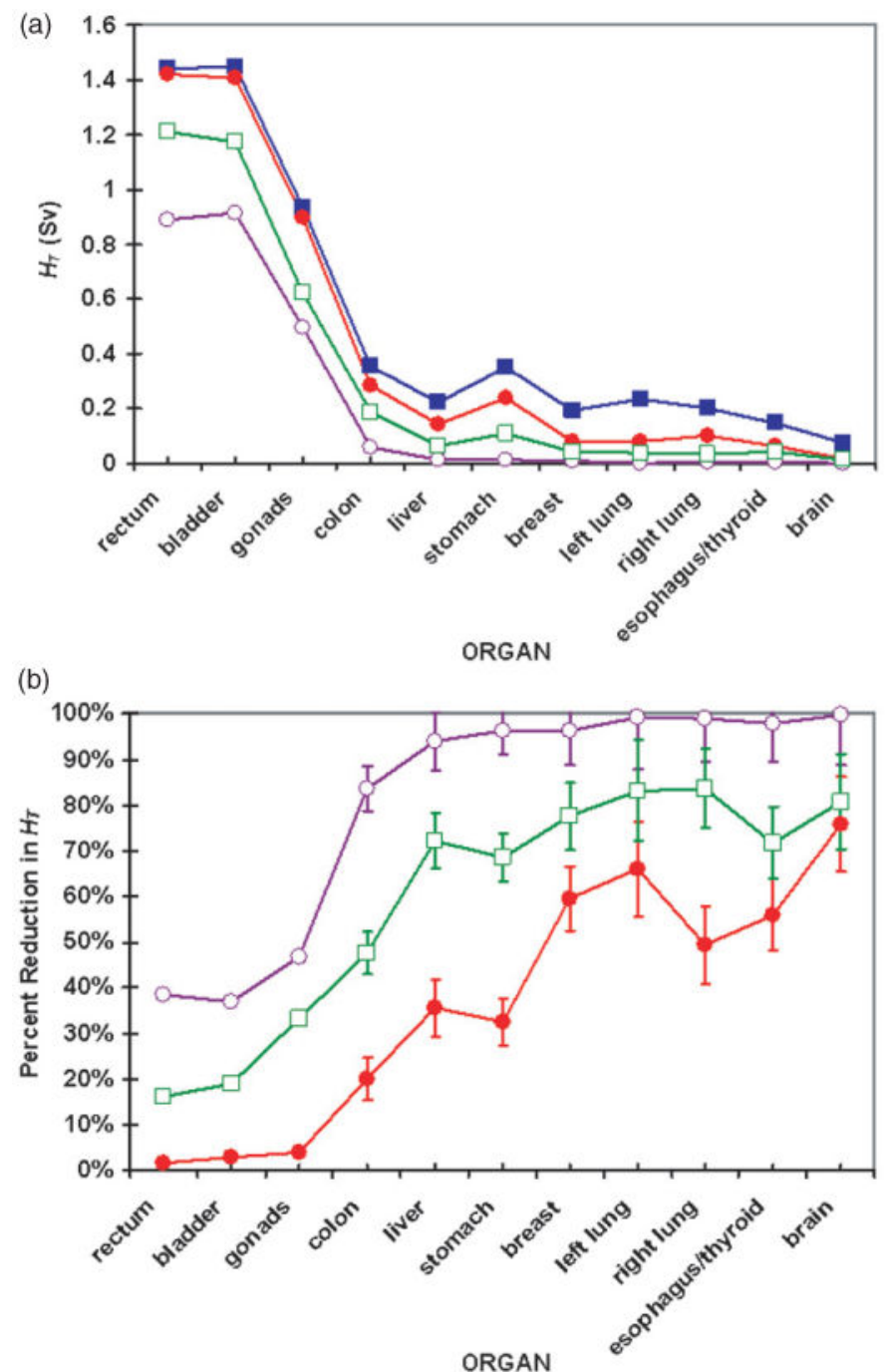

Figure 3.

(a) Equivalent dose from stray radiation, $H_{T}$, for each organ in simulations of the standard nozzle (mod0) (solid squares), a nozzle equipped with supplemental shielding and a tungsten-alloy final collimator $(\bmod 3)$ (solid circles), the equipped nozzle with an additional collimator far upstream of the patient (mod4) (open squares) and the ideal nozzle (mod5) (open circles). (b) Percent reduction in $H_{T}$ for each modified case. The lines in both (a) and (b) were added to guide the eye. Distance from the organ to isocenter increases from left to right on the plots. Error bars represent the $68 \%$ confidence interval. Where error bars are not shown, statistical uncertainties were contained within the data markers. 


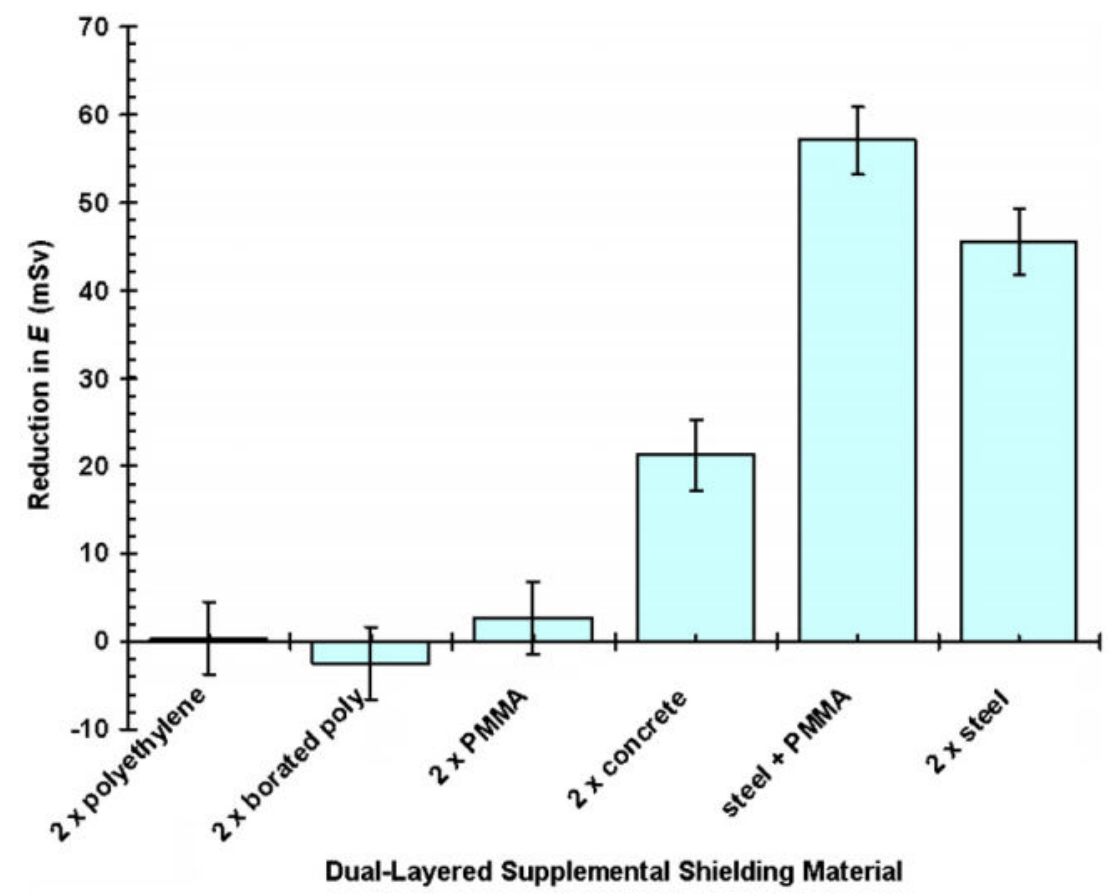

Figure 4.

Reduction in effective dose from stray radiation for various supplemental shielding materials for a 76 Gy SOBP proton treatment of the prostate. The supplemental shielding was added in two layers to the standard nozzle. For the 'steel + PMMA' case (mod2), the two supplemental layers were configured with the steel layer upstream of the PMMA layer. Error bars represent the $68 \%$ confidence interval. 


\section{Table 1}

Configurations of supplemental shielding that were added to the standard nozzle and evaluated with simulated SOBP treatments. The configurations were defined by the composition of the two layers of supplemental shielding. Layers 1 and 2 are shown as items E and F, respectively, in figure 1. Also listed are the masses of each dual-layer combination (PMMA is polymethyl methacrylate)

\begin{tabular}{llr}
\hline \multicolumn{2}{l}{ Supplemental shielding } & \\
\cline { 1 - 2 } Layer 1 & Layer 2 & Mass (kg) \\
\hline Polyethylene & Polyethylene & 307 \\
Borated polyethylene & Borated polyethylene & 311 \\
PMMA & PMMA & 389 \\
Concrete & Concrete & 765 \\
Steel & PMMA & 2067 \\
Steel & Steel & 2567 \\
\hline
\end{tabular}


$\pi \underset{\pi}{\pi} \frac{\mathscr{E}}{\mathrm{E}}$

言 $\frac{0}{\mathrm{~N}}$

$\frac{1}{N}$ वे

잉

흐 : ․ㅡ

ปี

胥 స్తే

离氞志

बิ $\frac{0}{2}$ क

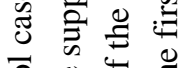

일 它

过专

N

产弯

켱 盯

芯 $\frac{0}{N}:$

$0 \cong$

$\exists \approx$

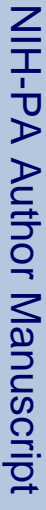

क⿺辶े

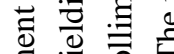

N

$\frac{0}{\circ}$

ปัँ

ज记

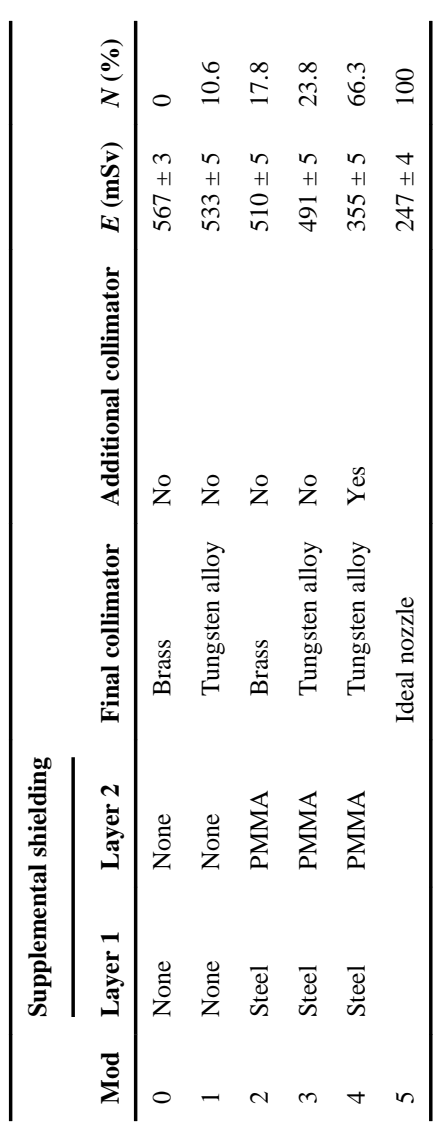

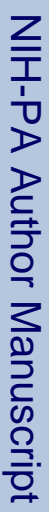

立高 元

कि के

实高哥

㝋氙

$\circ$ 흥

\%

4 N N

4 용

ธิ च ठ

氙 䒿

उ्ञाँ

त्ञ士

क

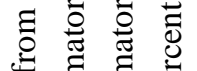

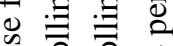

\% 용

可

昰田文

य ते

屯 స

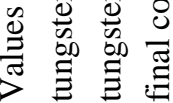

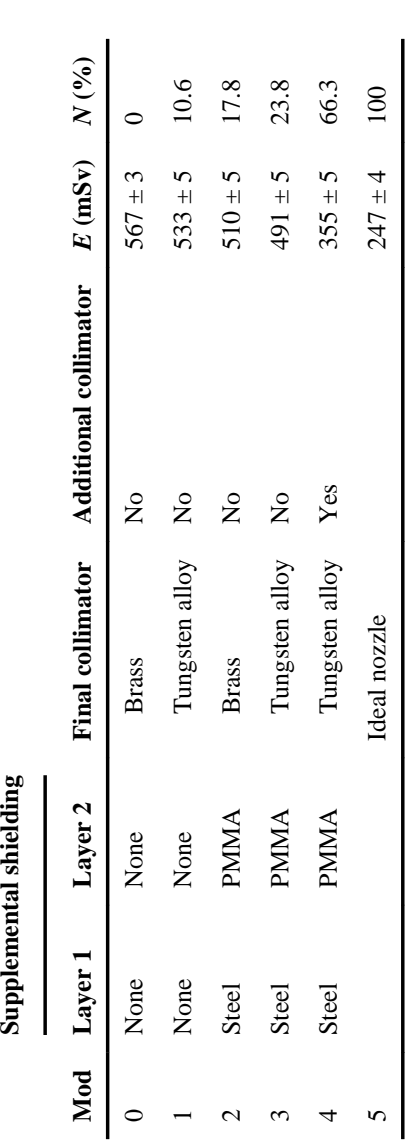

Phys Med Biol. Author manuscript; available in PMC 2014 August 26. 